\title{
Orbital Parameters of the Close Binary Central Stars of NGC 6337 and NGC 6026
}

\author{
Todd C. Hillwig ${ }^{1}$, Howard E. Bond ${ }^{2}$ and Melike Afsar ${ }^{3}$ \\ ${ }^{1}$ Department of Physics and Astronomy, Valparaiso University, Valparaiso, IN 46383, USA \\ email: todd.hillwig@valpo.edu \\ ${ }^{2}$ Space Telescope Science Institute \\ ${ }^{3}$ Ege University, Turkey
}

\begin{abstract}
We present the results of time-resolved photometry and spectroscopy of the central star of the planetary nebula NGC 6026 and time-resolved photometry of the central star of the planetary nebula NGC 6337. The results of period analysis give an orbital period of 0.528088 days for NGC 6026 and a photometric period of 0.173474 days for NGC 6337 . In the case of NGC 6337 it appears that the photometric period accurately reflects the orbital period and that the variability is the result of an irradiated hemisphere on the cool companion. For NGC 6026 however, radial velocities from spectroscopy show that the orbital period is twice the photometric period. In this case, the photometric variability is due to an ellipsoidal effect in which one of the stars fills, or nearly fills, its Roche lobe. Based on the data and modeling using the Wilson-Devinney code, we discuss the physical parameters of the two systems. We also relate the physical parameters to the shape and orientation of the nebulosity.
\end{abstract}

Keywords. binaries: close, planetary nebulae: individual (NGC 6337, NGC 6026), subdwarfs, white dwarfs

\section{Data and Orbital Parameters}

We used the Wilson-Devinney code (Wilson 1990; Wilson \& Devinney 1971) to model the close central stars (CSs) of the planetary nebulae NGC 6337 and NGC 6026. The modeling for NGC 6337 was limited due to the lack of a radial velocity curve leading to approximate limits for the physical parameters. For NGC 6026, the modeling included a single-line radial velocity curve, and led to a best-fit model to the data. Brief discussions of both systems follow.

\subsection{NGC 6337}

Our derived photometric ephemeris for inferior conjunction of the secondary is $T=$ 2452395.5368(1) $+0.1734742(5) E$, which agrees with that of Hillwig (2004).

The short orbital period (thus small separation) provides some useful constraints on model fitting: a secondary star with $M_{2}=0.35 M_{\odot}$ will have a main sequence radius larger than its Roche Lobe. We use this upper limit as a starting point for the secondary mass. The primary mass is set at the peak of the white dwarf mass distribution, $0.6 M_{\odot}$.

Lower values of $T_{C S}$ provide a good fit in a very narrow parameter space with values very close to those in model 1 (Figure 1a (dashed line) and Table 1).

For $T_{C S} \geqslant 90,000 \mathrm{~K}$, we are able to model the variability much more robustly. Inclination limits are found to be $10^{\circ} \leqslant i \leqslant 40^{\circ}$. Figure 1a (solid line) and Table 1 (Model 2) show the best-fit model at $i=9^{\circ}$. 

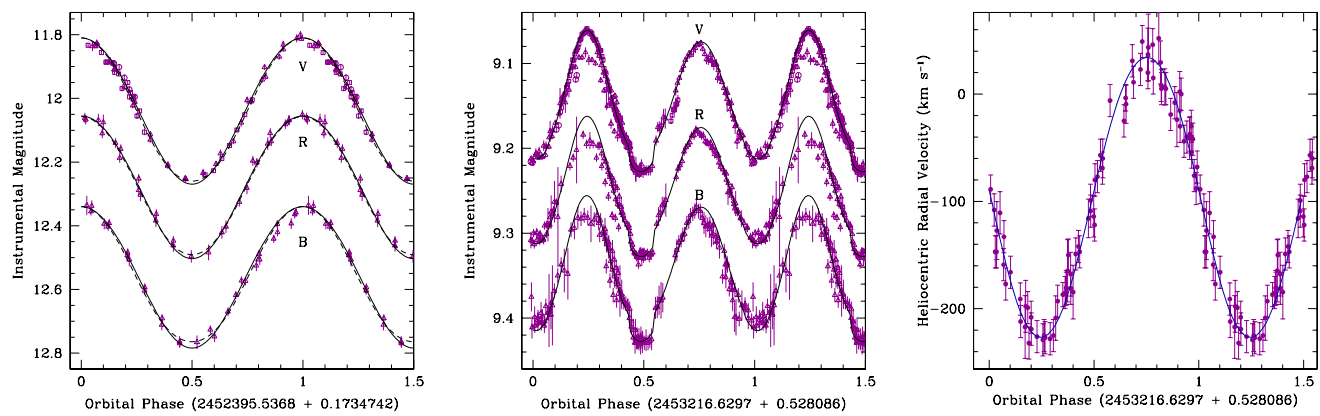

Figure 1. (a) The phase-folded V, R, and B light curves of NGC 6337. Also shown are the model fits discussed. Data are from 2002 (squares), 2003 (circles), and 2005 (triangles). The B data have been shifted for clarity. (b) The phase-folded V, R, and B-band light curves of NGC 6026. Data are from 2002 (squares), 2004 (circles), and 2005 (triangles). (c) The phase-folded radial velocity curve of NGC 6026

Table 1. Model Fitting Parameters

\begin{tabular}{lcccc|lccccc}
\hline & \multicolumn{2}{c}{ NGC 6337 } & \multicolumn{2}{c}{ NGC 6026 } & & \multicolumn{2}{c}{ NGC 6337 } & \multicolumn{2}{c}{ NGC 6026 } \\
Parameter & Model 1 & Model 2 & Model & Error & Parameter & Model 1 & Model 2 & Model & Error \\
\hline$T_{C S}\left(10^{3} \mathrm{~K}\right)$ & 45 & 105 & 36 & - & $R_{C S}\left(R_{\odot}\right)$ & 0.09 & 0.016 & 1.03 & 0.01 \\
$T_{2}\left(10^{3} \mathrm{~K}\right)$ & 5.5 & 2.3 & 134 & 5 & $R_{2}\left(R_{\odot}\right)$ & 0.42 & 0.34 & 0.053 & 0.005 \\
$M_{C S}\left(M_{\odot}\right)$ & 0.60 & 0.60 & 0.53 & 0.01 & $\log g_{C S}(\mathrm{cgs})$ & 6.30 & 7.78 & 4.14 & 0.02 \\
$M_{2}\left(M_{\odot}\right)$ & 0.35 & 0.20 & 0.53 & 0.01 & $\log g_{2}(\mathrm{cgs})$ & 4.74 & 4.67 & 6.72 & 0.02 \\
$\left.i^{\circ}\right)$ & 28 & 9 & 82 & 5 & $a\left(R_{\odot}\right)$ & 1.286 & 1.214 & 2.80 & 0.05 \\
$e$ & 0.0 & 0.0 & 0.025 & 0.002 & $\omega\left(^{\circ}\right)$ & - & - & 180 & 1 \\
\hline
\end{tabular}

\section{2. $N G C 6026$}

The CS spectrum is a good match to $15 \mathrm{Mon}$, an O7V star. Radial velocities were obtained through cross-correlation function (CCF) fitting. Radial velocities for the CS were used with the light curve data to determine a more accurate ephemeris. The derived photometric ephemeris superior conjunction of the CS is $T=2453216.6297(15)+0.528086(4) E$. Figure 1 shows the phase-folded light curves in all three observed filters. An apparent eclipse is seen at phase 0.5 .

The double-peaked light curve is likely caused by an ellipsoidal effect, with no strong irradiation variability. This suggests that the companion is likely a hot subdwarf/white dwarf due to the apparent eclipse at phase 0.5, but lack of an obvious eclipse at phase 0.0. The results of the models are shown with the data in Figure 1 and in Table 1. The best-fit model suggests an eccentric orbit. What appear to be intrinsic variations in the photometry from night-to-night complicate the fitting.

\section{References}

Hillwig, T. C. 2004, in M. Meixner, J. H. Kastner, B. Balick \& N. Soker (eds.), Asymmetrical Planetary Nebulae III: Winds, Structure and the Thunderbird, ASP Conf. Series (San Francisco: ASP), vol. 313 , p. 529

Wilson, R.E. 1990, ApJ, 356, 613

Wilson, R.E., \& Devinney, E. J. 1971, ApJ, 166, 605 